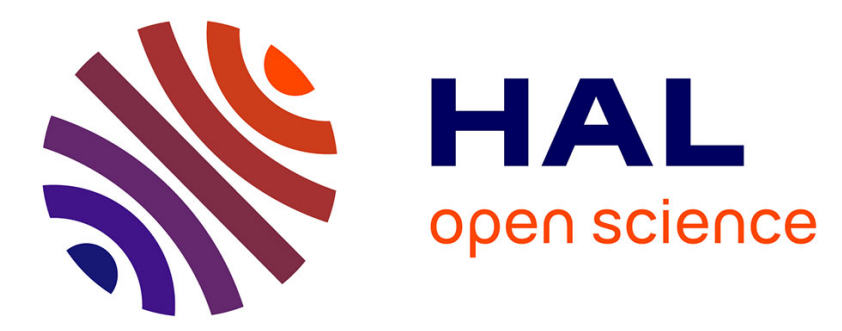

\title{
Des douleurs abdominales de cause vasculaire
}

\author{
A. Nuzzo, M. Ronot, L. Maggiori, F. Joly, O. Corcos
}

\section{To cite this version:}

A. Nuzzo, M. Ronot, L. Maggiori, F. Joly, O. Corcos. Des douleurs abdominales de cause vasculaire. La Revue de Médecine Interne, 2019, 40, pp.129 - 131. 10.1016/j.revmed.2017.12.001 . hal-03484614

\section{HAL Id: hal-03484614 \\ https://hal.science/hal-03484614}

Submitted on 20 Dec 2021

HAL is a multi-disciplinary open access archive for the deposit and dissemination of scientific research documents, whether they are published or not. The documents may come from teaching and research institutions in France or abroad, or from public or private research centers.
L'archive ouverte pluridisciplinaire HAL, est destinée au dépôt et à la diffusion de documents scientifiques de niveau recherche, publiés ou non, émanant des établissements d'enseignement et de recherche français ou étrangers, des laboratoires publics ou privés.

\section{다)(1) $(5$}

Distributed under a Creative Commons Attribution - NonCommercial| 4.0 International 


\section{Des douleurs abdominales de cause vasculaire Abdominal pain of vascular cause}

A. Nuzzo $(1,2,5)^{*}$, M. Ronot $(1,3,5)$, L. Maggiori $(1,4,5)$, F. Joly $(1,2,5)$, O. Corcos $(1,2,5)$

1. Structure d'URgences Vasculaires Intestinales (SURVI), Hôpital Beaujon, APHP, 100 boulevard du Général Leclerc 92110 Clichy, France

2. Service de Gastroentérologie, MICI, Assistance nutritive, Hôpital Beaujon, APHP, 100 boulevard du Général Leclerc 92110 Clichy, France

3. Service de Radiologie, Hôpital Beaujon, APHP, 100 boulevard du Général Leclerc 92110 Clichy, France

4. Service de Chirurgie Colorectale, Hôpital Beaujon, APHP, 100 boulevard du Général Leclerc 92110 Clichy, France

5. Université Paris Diderot, Sorbonne Paris Cité, Paris, France

\section{*Auteur correspondant}

A. Nuzzo, SURVI (Structure d'URgences Vasculaires Intestinales), Service de Gastroentérologie, MICI, Assistance nutritive, Hôpital Beaujon, Clichy, France, Université Paris VII (Denis Diderot), 100 boulevard du Général Leclerc, 92110 Clichy, France (Tel: +33 1408756 16; Fax: +33 1408745 74;

E-mail: al.nuzzo@gmail.com

Mots clés : ischémie mésentérique aiguë; ischémie intestinale; colite ischémique; nécrose intestinale; scanner

Key words: acute mesenteric ischemia; intestinal ischemia; colon ischemia; intestinal necrosis; computed tomography 


\section{L'histoire}

Un homme, âgé de 29 ans, consultait aux urgences pour une douleur abdominale brutale, intense, diffuse et inhabituelle. Ce patient sans antécédent médical rapportait une consommation de tabac et de cannabis régulière. L'examen physique était sans particularité en dehors d'une obésité de grade 1, et ne retrouvait pas de fièvre ni distension abdominale ni signe d'irritation péritonéale notamment. Les examens biologiques montraient une hyperleucocytose à $24 \mathrm{G} / \mathrm{L}$, une $\mathrm{CRP}$ à $138 \mathrm{mg} / \mathrm{L}$, une créatininémie et un lactate sérique normaux. La douleur n'était pas soulagée par les antalgiques de palier II. Un scanner abdomino-pelvien avec injection de produit de contraste était réalisé en urgence (fig. 1).

\section{Le diagnostic}

Une ischémie mésentérique aiguë précoce.

\section{Les commentaires}

Dans cette observation, le diagnostic n'avait pas été posé immédiatement devant l'absence de signe de souffrance digestive sur le scanner initial et en dépit de l'occlusion de l'ensemble du réseau artériel digestif. Le patient était traité par une héparine non fractionnée à dose curative, un antalgique morphinique, et surveillé en hospitalisation conventionnelle de chirurgie. Après 48 heures, son état clinique se dégradait avec l'apparition d'une fièvre, d'une distension abdominale puis d'une hypotension artérielle. Le lactate sérique s'élevait alors à 5 $\mathrm{mmol} / \mathrm{L}$ (normal $<2 \mathrm{mmol} / \mathrm{L}$ ). Le deuxième scanner retrouvait une dilatation sans obstacle de l'ensemble de l'intestin grêle associée à un défaut de rehaussement pariétal (fig. 2) évocateur d'ischémie mésentérique aiguë tardive au stade de nécrose (infarctus mésentérique). Une laparotomie en urgence était proposée retrouvant un infarctus de tout l'intestin grêle et des côlons droit et transverse indiquant à une résection étendue du duodénum à l'angle colique 
gauche (fig. 3). Le patient était ensuite transféré pour insuffisance intestinale et assistance nutritive, lié à un syndrome du grêle court. L'enquête diagnostique étiologique retrouvait un syndrome des anti-phospholipides. Après réhabilitations nutritionnelle (nutrition parentérale totale), vasculaire (pontage coliaque et de l'artère mésentérique inférieure, aspirine et héparine) et digestive (anastomose duodéno-colique), le patient a pu retrouver une alimentation orale et rentrer à domicile avec une nutrition parentérale quotidienne nocturne.

Ce cas représente une situation d'ischémie mésentérique aiguë authentique, vue très précocement, au cours de laquelle les examens cliniques, biologiques mais aussi radiologiques ont été initialement confondants et ont contribué à un retard diagnostique et thérapeutique. Ceci est d'autant plus problématique qu'à ce stade très précoce, l'ischémie mésentérique aiguë est potentiellement totalement réversible par un traitement urgent et spécifique incluant une revascularisation [1]. De plus, ce cas confirme l'évolution naturelle de l'ischémie mésentérique aiguë en 2 stades, précoce et tardif, au cours desquels les signes de nécrose intestinale sont respectivement absents puis présents [2,3]. Nous avons récemment suggéré au sein d'une étude prospective qu'une laparotomie devait être discutée en urgence en présence d'au moins un des 3 facteurs prédictifs de nécrose intestinale transmurale irréversible caractéristiques du stade tardif de l'affection (présence d'une défaillance d'organe, lactate $>2 \mathrm{mmol} / \mathrm{l}$, dilatation intestinale $>2,5 \mathrm{~cm}$ sur le scanner) [2].

L'originalité de cette observation repose sur le fait que, derrière la normalité apparente des anses digestives sur le scanner initial, le diagnostic constituait une grande urgence vitale et digestive. L'apparition retardée des signes de souffrance intestinale sur le scanner a déjà été rapportée chez un patient ayant une ischémie mésentérique non-occlusive et chez lequel le diagnostic avait été confirmé par une imagerie de «second-look» 12 heures plus tard [4]. En l'absence de biomarqueur diagnostique actuellement disponible en pratique clinique, il est important de rappeler la définition de l'ischémie mésentérique aiguë qui associe des signes 
cliniques, biologiques et/ou radiologiques 1) d'insuffisance vasculaire et 2) de souffrance digestive $[3,5]$. Le diagnostic précoce repose sur un haut degré de suspicion par les cliniciens et chirurgiens devant toute douleur abdominale brutale, inhabituelle et dont la sédation nécessite des antalgiques majeurs. Une telle présentation doit conduire à la réalisation en urgence d'un scanner abdomino-pelvien sans et avec injection de produit de contraste aux temps artériel et portal, permettant une analyse spécifique de la vascularisation digestive et des signes de souffrance digestive [5].

\section{Conflits d'intérêts : aucun}

\section{Figures}

Fig. 1. Premier angioscanner abdomino-pelvien initial : occlusion proximale du tronc cœliaque et de l'artère mésentérique supérieure (flèches) en coupe sagittale (A), anses digestives d'aspect normales (têtes de flèches) en coupe axiale (B), sans signe radiologique de souffrance digestive (pas d'épaississement ni défaut de rehaussement pariétal gastro-intestinal ou colique).

Figure 2. Deuxième angioscanner abdomino-pelvien à $48 \mathrm{~h}$ : dilatation marquée avec défaut de rehaussement pariétal de l'ensemble de l'intestin grêle évocateur d'un infarctus intestinal étendu (têtes de flèches)

Figure 3. Troisième angioscanner, post-opératoire : mésentère «fantomatique » sans intestin grêle (têtes de flèches)

\section{Références}


1 Corcos O, Castier Y, Sibert A, Gaujoux S, Ronot M, Joly F, et al. Effects of a multimodal management strategy for acute mesenteric ischemia on survival and intestinal failure. Clin Gastroenterol Hepatol 2013;11:158-65 e2.

2 Nuzzo A, Maggiori L, Ronot M, Becq A, Plessier A, Gault N, et al. Predictive factors of intestinal necrosis in acute mesenteric ischemia: prospective study from an intestinal stroke center. Am J Gastroenterol 2017;112:597-605.

3 Nuzzo A, Corcos O. L'ischémie mésentérique à l'ère des structures d'urgences vasculaires intestinales. Rev Med Interne 2017;38:592-602

4 Arunachalam K. Stuttering mesenteric ischemia. Am J Med 2016;129:e25-6.

5 Menke J. Diagnostic accuracy of multidetector CT in acute mesenteric ischemia: systematic review and meta-analysis. Radiology 2010;256:93-101. 


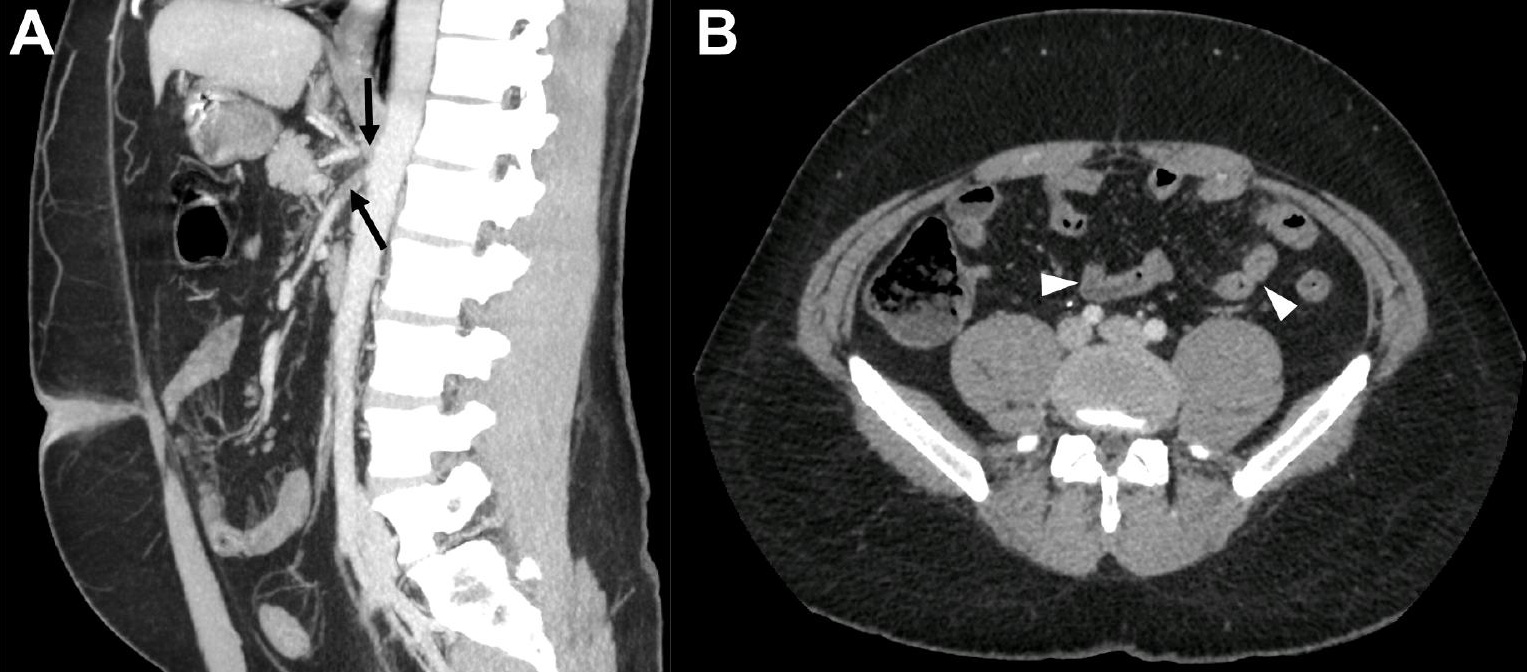




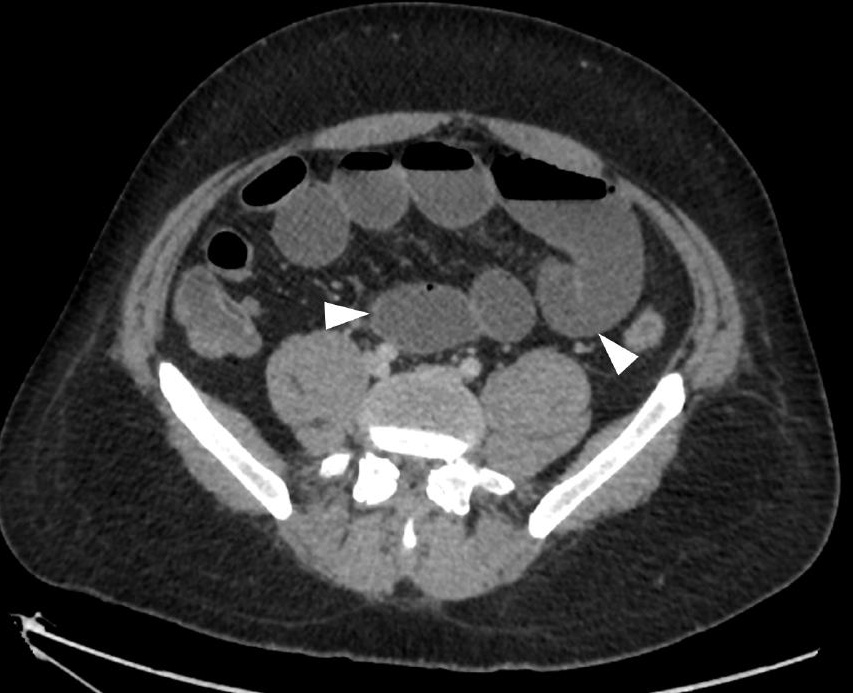




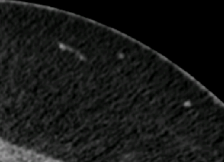

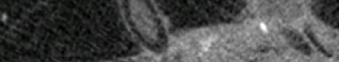

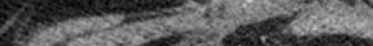

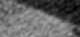

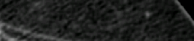

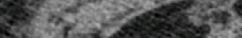

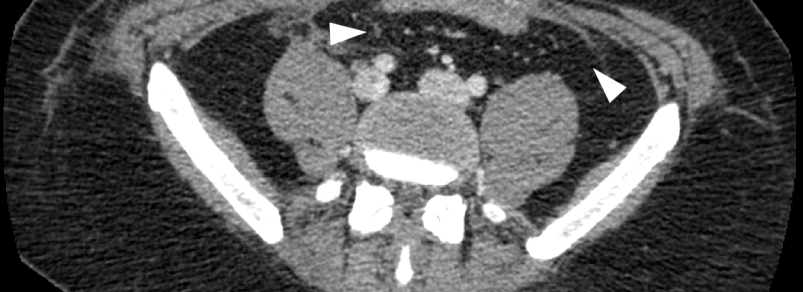

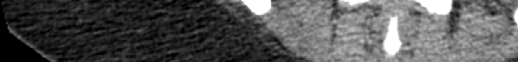

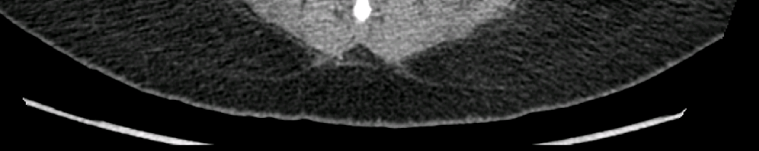

